\title{
Indicadores de Qualidade em Indústria Farmacêutica
}

\author{
Quality Indicators in Pharmaceutical Industry
}

Recebido em: 22/07/2017

Aceito em: $23 / 10 / 2017$
Ana Débora Nunes Pinheiro

Programa de Pós-Graduação em Química, Instituto de Química, Universidade Federal Fluminense. Campus Valonguinho, Outeiro de São João Batista, s/n, Centro, CEP 24020-141. Niterói, Rio de Janeiro, Brasil.

E-mail: anadebora@gmail.com

\section{ABSTRACT}

The quality of pharmaceutical industrial processes - and how to achieve it - is a theme of great interest on the pharmaceutical industry, especially in what concerns its standardization. With the standardization of the practices and the normatization of the good manufacturing guidelines, a concern it started, also from the government, with what would be quality in the pharmaceutical industry and how to achieve it. This work aims to gather main guidelines and practices used as quality indicators in the pharmaceutical industry. Therefore, it was selected 21 review and research articles obtained through a search on the online databases, as well reference guidelines from international organizations, such as World Health Organization (WHO), Food and Drug Administration (FDA) and International Council for Harmonization of Technical Requirements for Pharmaceuticals for Human Use (ICH). After organizing and studying the selected material, the quality practices currently used in the pharmaceutical industry was described, such as quality risk management, quality by design, corrective and preventive actions, lean manufacturing, total quality system and ISO series. Although a complete and updated published regimentation about quality indicators and practices in the pharmaceutical industry is already available, the publications lack specific information and practical experiences on the implementation of these quality systems, being hard to exemplify the positive influence of these strategies on the management of pharmaceutical industries.

keywords: pharmaceutical industry; quality indicators; quality systems

\section{RESUMO}

A qualidade de processos industriais farmacêuticos e como obtê-la é um tema de grande interesse na indústria farmacêutica, principalmente no que diz respeito a sua padronização. Com a normatização dos guias de boas práticas farmacêuticas e a padronização das práticas, passou a ocorrer uma maior preocupação, inclusive governamental, com o que seria qualidade e como alcançá-la. O objetivo deste trabalho foi reunir os principais guias de referência e as práticas utilizadas como indicadores de qualidade na indústria farmacêutica. Para tanto, foram selecionados 21 trabalhos obtidos por meio de pesquisa em bancos de dados disponíveis online, além de guias de referência de organizações internacionais, como Organização Mundial da Saúde (OMS), Food and Drug Administration (FDA) e International Council for Harmonization of Technical Requirements for Pharmaceuticals for Human Use (ICH). Após a organização e a leitura do material selecionado, foram descritas algumas práticas de qualidade atualmente utilizadas na indústria farmacêutica: gestão de risco, quality by design, ações corretivas e preventivas, lean manufacturing, sistema de qualidade total e séries ISO. Apesar de existir uma regulamentação bastante completa e atual acerca de indicadores e práticas de qualidade em indústria farmacêutica, as publicações não relatam informações específicas e experiências práticas da implementação desses sistemas de qualidade, dificultando assim, exemplificar melhor como essas estratégias influenciam positivamente no gerenciamento das indústrias farmacêuticas.

Palavras-chave: indústria farmacêutica; indicadores de qualidade; sistemas de qualidade. 
INTRODUÇÃO

$\mathrm{Na}$ indústria farmacêutica, a qualidade é um tema de extrema importância, pois existe uma grande preocupação por parte desse setor para que o sistema de gestão da qualidade funcione de forma a proporcionar produtos fabricados de qualidade e também garantir a satisfação dos consumidores. O controle e a gestão da qualidade nos segmentos industriais são exigências de normas e legislações governamentais (1). Neste sentido, o papel dos governos passou a se destacar após acordos firmados entre a Federação Farmacêutica Internacional (FIP) e a Federação Internacional das Associações de Indústrias Farmacêuticas (IFPMA) (2).

O Sistema de Gestão da Qualidade também é conhecido como Total Quality Management (TQM) ou Sistema de Qualidade Total (SQT). Esse sistema funciona como uma estratégia que tem como finalidade gerir, orientar e conscientizar os setores da empresa, seja indústria ou não, visando a qualidade dos processos e, no final, a aprovação do cliente (3). Para que o TQM seja efetivo e eficaz, existem técnicas que demonstram numericamente o índice de qualidade e seus resultados na indústria farmacêutica. Essas técnicas são conhecidas como os indicadores da qualidade.

De uma forma geral, tudo que acontece na indústria deve ser controlado e documentado. O setor responsável por esse gerenciamento é a garantia da qualidade, que, por sua vez, é fiscalizado pela Agência Nacional de Vigilância Sanitária (Anvisa). A Anvisa é o órgão governamental que faz cumprir os requisitos legais no setor farmacêutico; além disso é o órgão brasileiro que expede o Certificado de Boas Práticas de Fabricação (BPF), ou Good Manufacturing Practices (GMP), necessário para que a indústria possa produzir medicamentos (4).

No Brasil, a indústria de medicamentos deve seguir os requisitos especificados na Resolução da Diretoria Colegiada no17/2010 (RDC 17/2010) (4) publicada pela Anvisa. Além desta RDC, existem outros documentos utilizados, como alguns guias de órgãos internacionalmente conhecidos e respeitados. Os guias de referência internacionais mais amplamente difundidos, e por isso mais utilizados na indústria farmacêutica, são os guias da Organização Mundial de Saúde (OMS), do International Concil for Harmonization (ICH) e Food and Drug Administration (FDA). Além disso, as empresas podem aderir voluntariamente às normas de certificação da International Stadardization Organization (ISO), por meio de uma empresa certificadora.
Esse trabalho tem como objetivo reunir e discutir os principais guias regulamentados e as práticas utilizadas como indicadores da qualidade na indústria farmacêutica.

\section{MÉTODOS}

O levantamento e a seleção dos artigos foram realizados na internet, nos bancos de dados Science Direct, Web of Knowledge e SciELO. A pesquisa foi realizada utilizando busca pelos descritores "pharmacentical industry" com refinamento de "quality" e/ou "quality indicator(s)" e/ou "quality practice(s)" para periódicos internacionais e "qualidade na indústria farmacêutica" para o banco de dados SciELO. Os critérios de exclusão, bem como o período relacionado às datas de publicação, podem ser visualizados na Tabela 1 .

Os critérios utilizados para a seleção da amostra foram: artigos publicados eletronicamente, nos idiomas inglês ou português, que abordassem a temática Indicadores de Qualidade na Indústria Farmacêutica. Devido ao número de publicações disponíveis no banco de dados Science Direct, foi priorizado, para a seleção dos artigos, o período de 2006 a 2017, representando assim os últimos 11 anos. Também foram incluídos neste trabalho livros e capítulos de livro disponíveis nos bancos de dados utilizados. Foram excluídos do estudo: editoriais, cartas ao leitor e estudos que não abordassem a temática relevante aos objetivos desta revisão.

Este trabalho contou com a seleção de 34 publicações, as quais estão relacionadas com indicadores de qualidade na indústria farmacêutica e com práticas de qualidade em geral. Além disso, foram levados em consideração 13 guias de referência das seguintes organizações: Anvisa, OMS, FDA e ICH.

O resultado do compilamento dos materiais utilizados nesse estudo foi analisado de forma descritiva.

\section{RESULTADOS E DISCUSSÃO}

Guias de referência para gerenciamento da qualidade em indústria farmacêutica

Guia Nacional. No Brasil, a Anvisa publicou uma norma de boas práticas farmacêuticas para medicamentos, a RDC n ${ }^{\circ} 17$, de 16 de abril de 2010, que tem como objetivo estabelecer os requisitos mínimos para a fabricação de medicamentos a fim de padronizar a verifica- 
ção do cumprimento das boas práticas de fabricação de medicamentos de uso humano (4). Em 2015 foi feita uma atualização desta norma, sendo publicada no Diário Oficial da União a RDC 33/2015, a qual atualiza as normas para fabricação de produtos não farmacêuticos e para produtos que não estão sujeitos à vigilância sanitária (5). A RDC 33/2015 alterou o artigo 215 da RDC $17 / 2010$, onde constam as normas de compartilhamento de áreas produtivas. Com a atualização da RDC, passou a ser permitido que produtos de uso veterinário possam ser fabricados nas mesmas instalações que medicamen- tos para uso humano, contanto que todos os insumos sejam aprovados para uso humano e desde que haja pelo menos um medicamento já comercializado com tais insumos. Além disso, produtos de saúde, higiene, cosméticos e alimentos também podem ser produzidos, caso haja comprovação técnica de que seus insumos sejam de qualidade igual ou superior àqueles usados para medicamentos. Também se faz necessário que, neste caso, sejam cumpridos os requisitos de boas práticas de fabricação da RDC 17/2010 e as Boas Práticas de Fabricação (BPF) específicas para a categoria.

Tabela 1. Bancos de dados e critérios utilizados para seleção de artigos utilizados na pesquisa

\begin{tabular}{|c|c|c|c|c|c|}
\hline Base de dados & Busca & Refinamento & Exclusão 1 & Exclusão 2 & $\begin{array}{c}\text { Itens } \\
\text { selecionados }\end{array}$ \\
\hline \multirow{2}{*}{ Web of Knowlegde } & \multirow{2}{*}{$\begin{array}{l}\text { pharmaceutical } \\
\text { industry }(n=226)\end{array}$} & Quality ( $n=88)$ & Área de pesquisa $(n=15)$ & $\begin{array}{l}\text { Publicação em livros, congressos, } \\
\text { apenas resumo e assunto do artigo }\end{array}$ & 6 \\
\hline & & $\begin{array}{l}\text { Quality e quality } \\
\text { indicators ( } n=8)\end{array}$ & Assunto $(n=2)$ & - & 2 \\
\hline Scielo.org & $\begin{array}{l}\text { gestão da qualidade } \\
\qquad(\mathrm{n}=51)\end{array}$ & Indústria $(\mathrm{n}=8)$ & Assunto $(n=2)$ & - & 2 \\
\hline \multirow{2}{*}{ Science Direct } & $\begin{array}{l}\text { pharmaceutical } \\
\text { industry + quality } \\
\text { indicators ( } n=13)\end{array}$ & $\begin{array}{c}\text { Ano de publicação } \\
\begin{array}{c}(2006-2017) \\
(n=11)\end{array}\end{array}$ & Assunto $(n=0)$ & - & 8 \\
\hline & $\begin{array}{l}\text { gestão da qualidade } \\
\qquad(\mathrm{n}=2009)\end{array}$ & $\begin{array}{l}\text { Ano de publicação } \\
\begin{array}{c}(2006-2017) \\
(n=1602)\end{array}\end{array}$ & $\begin{array}{l}\text { Assunto }(n=20) \text { e Área de } \\
\text { pesquisa }(n=15)\end{array}$ & - & 3 \\
\hline
\end{tabular}

Além de medicamentos de uso humano, a Anvisa também regulamentou a fabricação de outros produtos utilizados para a saúde (6). Em 2013, essa agência publicou a RDC 13/2013 (6) que dispõe sobre as boas práticas de fabricação de produtos tradicionais fitoterápicos; e em 2009, com a publicação da RDC 63/2009 foram estabelecidas as boas práticas de fabricação de radiofármacos (7).

Em 2008, foi publicada a RDC 69/2008 (8) que dispunha sobre as boas práticas de fabricação de gases medicinais, com atualização em 2010 da publicação da RDC 9/2010 (9), que estabeleceu a abrangência do regulamento técnico sobre as BPF de gases medicinais. Dessa forma, o regulamento passou a se aplicar, além das empresas de produção, às que, embora não participem do processo completo, elaborem alguma etapa do processo fabril, como envase, tanques criogênicos e caminhões-tanque. Além disso, regulamentou o prazo para autorização de funcionamento para obtenção do certificado de BPF.

Guias Internacionais. Os guias internacionais mais amplamente difundidos, e por isso mais utilizados na indústria farmacêutica, são aqueles publicados pela OMS, ICH e FDA. Os guias da Anvisa são formulados com especificações para a indústria brasileira, visando atender as necessidades internas do país. O mesmo vale para o FDA nos Estados Unidos da América (EUA). Entretanto, os guias nacionais acabam sendo fortemente influenciados pelos guias de organizações reconhecidas e respeitadas internacionalmente, sendo eles adaptados à realidade de cada país.

Guia OMS. A Organização Mundial da Saúde publicou, em 2004, uma coletânea sobre boas práticas de fabricação intitulada Quality Assurance of Pharmaceuticals. Essa publicação reúne guias e materiais relacionados à manutenção da qualidade de produtos farmacêuticos. O volume dois dessa publicação diz respeito às boas práticas de fabricação e inspeção (10).

Guia ICH. A fim de criar discussões científicas e técnicas sobre todos os aspectos regulatórios de medicamentos, proporcionando assim produtos seguros, efetivos e de alta qualidade que são desenvolvidos e registrados da forma mais eficiente possível (11), em 1990 foi criado o International Council for Harmonization of Technical (11), pelo ICH. O ICH é um projeto especial que reúne autoridades europeias, japonesas e norte-americanas, além de peritos da indústria farmacêutica das 
três regiões participantes. O objetivo de tal projeto é a utilização mais eficiente dos recursos humanos, animais e materiais, além de excluir o que não for essencial e que possa estar causando atrasos no desenvolvimento e na disponibilidade de novos medicamentos e, ao mesmo tempo, resguardar a qualidade, segurança, eficácia e obrigações regulatórias para proteger a saúde pública (11).

Em abril de 2009, a ICH publicou um guia para a indústria, o Q10 Pharmaceutical Quality System (12), que visa auxiliar as indústrias farmacêuticas por meio da descrição de um modelo de sistema gerencial efetivo, denominado sistema de qualidade farmacêutico ou modelo ICH Q10.

O ICH Q10 descreve um modelo de sistema que é baseado nos conceitos da ISO, incluindo conceitos de qualidade, normas de boas práticas de fabricação, além de complementar outros guias ICH, como ICH Q8 pharmaceutical development (13) - e ICH Q9 - quality risk management (14). O ICH Q10 descreve um sistema que pode ser implementado em diferentes etapas do ciclo de produção de um produto (12). A maior parte da normatização deste guia também está especificada em guias regionais de boas práticas de fabricação, como o da Anvisa (4). A ideia do ICH Q10 é complementar as normas já existentes, portanto o cumprimento das etapas descritas exclusivamente neste guia é opcional para as indústrias. É interessante ressaltar que esses guias sofrem atualizações frequentes.

A implementação desse guia no ciclo de produção de um medicamento, propõe facilitar a inovação e a melhora continuada, além do fortalecimento da ligação entre o desenvolvimento farmacêutico e as atividades industriais.

A Anvisa passou a integrar o ICH (15), de forma que irá contribuir significativamente para o desenvolvimento e aprovação dos guias ICH. Além disso, a agência deverá se adequar aos guias ICH até o final de 2021.

Guia FDA. O FDA é o órgão governamental norte-americano responsável pela regulamentação de alimentos, medicamentos, cosméticos, saneantes e correlatos nos EUA (16).

Com relação às boas práticas de fabricação, em 2002 a FDA anunciou o projeto Pharmaceutical cGMP for the 21st Century - A risk-based approach (17) que possuía como objetivo integrar as abordagens de sistema de qualidade e gerenciamento de risco a fim de que a indústria fosse encorajada a adotar tecnologias de fabricação novas e modernas.
Em 2006, foi publicado o guia Quality Systems Approach to Pharmaceutical cGMP Regulations (18), que discorre sobre a implementação de um sistema de qualidade moderno com abordagem de gerenciamento de riscos, a fim de atender os requisitos da própria FDA com relação à regulação das boas práticas de fabricação. Além disso, esse guia descreve um modelo de qualidade de sistema, com destaque para um modelo consistente com os requisitos de BPF para medicamentos de uso humano e veterinário (18).

\section{Práticas aplicadas e relacionadas ao gerenciamento da qualidade em indústria farmacêutica}

Um estudo realizado em 2004 identificou que algumas das práticas de gestão da qualidade em empresas do segmento farmacêutico são: gestão de risco, quality by design, ações corretivas e preventivas, séries ISO (19); e, mais recentemente, práticas de outros tipos de indústria também vêm sendo aplicadas, como lean manufacturing (indústria automobilística, p.ex. Toyota), sistema de qualidade total e six sigma (indústria tecnológica, p.ex. Motorola, General Electric).

Gestão de risco. A gestão de risco é uma ferramenta utilizada para identificar riscos e é definida como um método para avaliação, controle, comunicação e revisão de riscos para a qualidade do produto (20). Essa estratégia permite que a empresa atue de forma preventiva, eliminando possíveis perdas. A gestão de riscos é um processo contínuo e deve ser aplicado quando $(20,21)$ : a) houver necessidade de implementar controles não previstos inicialmente; b) um novo trabalho for planejado; c) existirem regulamentos técnicos e legais e suas modificações.

Além disso, esse processo também deve ser aplicado periodicamente, envolvendo atividades rotineiras, emergenciais e futuras (21).

Quality by design. O termo quality by design foi criado tendo como pensamento que um produto a ser desenvolvido deve levar em consideração qualidade, segurança e efetividade. Neste processo, a cada etapa, a qualidade deve ser testada, no sentido de aumentar as probabilidades de o produto final possuir as especificações planejadas inicialmente (21). A qualidade não deve ser testada no estágio final do processo de fabricação, mas construída ou pré-definida por meio do design do produto $(21,22,13)$.

A aplicação dos princípios do quality by design tem sido de interesse da indústria farmacêutica, com alvo em produtos biofarmacêuticos (22-24). 
Ações corretivas e preventivas. As ações preventivas e corretivas possuem como finalidade melhorar continuamente o desempenho dos processos em uma empresa. Uma vez que prevenir geralmente é mais barato do que implementar ações que visem corrigir um problema, utilizar a estratégia de ações preventivas pode significar redução de custos (25). Por outro lado, as ações corretivas são práticas necessárias para que um problema ocorrido não traga novos problemas. As ações corretivas são eficazes somente quando medidas são tomadas imediatamente após o inconveniente ter ocorrido, de forma a amenizar suas consequências $(25,26)$.

Para implementar essas ações, na maioria das vezes, se adota o PDCA (26) - plan, do, check e act ou seja, planejar, executar, verificar e agir. O PDCA descreve como as mudanças devem ocorrer, de acordo com a verificação, há um planejamento e uma implementação de mudança com a consequente melhoria almejada. Essa técnica também pode ser denominada Ciclo de Deming (27).

Six sigma. Outra ferramenta utilizada para identificar e implementar melhorias nos processos internos de uma empresa é o Sig sigma, que possui a finalidade de assegurar custos operacionais menores com consequente aumento de lucros. O six sigma foi originado do Sistema de Qualidade Total (SQT) utilizando os conceitos de que a qualidade é de responsabilidade de todos, com foco na satisfação dos clientes e significativo investimento na educação e treinamento em estatística, análise de causa raiz, e metodologias de solução de problemas $(27,28)$.

A estratégia six sigma pode ajudar a empresa a reduzir erros e aumentar a rentabilidade utilizando vários princípios básicos (29), que são definir, medir, analisar, melhorar e controlar $(25,28)$. Essa ferramenta é utilizada para calcular matematicamente o nível de desempenho dos processos e obter um diagnóstico. A escala de medida vai de $1-\sigma$ a $6-\sigma$, sendo este último o mais alto nível de excelência.

Lean manufacturing. Com o objetivo de reduzir o consumo de recursos que não agregavam valor aos produtos da empresa (30), foi criado o lean manufacturing (ou sistema Toyota de produção, ou produção just-in-time). Esse sistema identifica e elimina atividades sem valor agregado, com um processo de melhoria contínua e fluxo de material por meio do pedido do cliente (29).

A essência dessa prática é reduzir os desperdícios da indústria, que ocorrem em espera, defeito, transporte, movimentação, excesso de estoque e de produto acabado, mau ou super processamento.
Seguindo esse princípio de redução de desperdício, passa a ocorrer menos gastos, menor tempo de design, menos camadas organizacionais e menos fornecedores com mais emprego de funcionários, além de uma maior flexibilidade e capacidade, levando assim, a empresa, a uma maior produtividade e menor insatisfação dos clientes. E isso leva à competitividade de sucesso (31).

Sistema de Qualidade Total. O Sistema de Qualidade Total (SQT) é uma adaptação do Total Quality Control (TQC) que foi desenvolvido na década de 1950 no Japão, logo após a $2^{a}$ guerra mundial (32). O SQT é baseado em conjuntos de conceitos, práticas e ferramentas de gestão que foram empregados pela maioria das boas empresas ao redor do mundo.

O SQT é um conceito sistemático, integrado e que possui perspectiva consistente que envolve tudo que está relacionado a uma empresa (3), além de implicar em envolvimento total de uma empresa. A utilização desse sistema visa a racionalização da gestão de um sistema complexo, sendo que a eficiência, neste caso, não está relacionada a apenas um elemento (32). O SQT é descrito como uma filosofia de gerenciamento que na qual a organização que é movida pela satisfação do cliente por meio de melhoria contínua na eficiência e eficácia da empresa e dos processos relacionados a ela. Esse sistema tem como principais características a melhoria da qualidade e da performance de robustez, produtividade, relacionamento entre clientes e fornecedores e envolvimento de todos os colaboradores e orientação objetiva para o mercado (3).

Série ISO. A ISO é uma organização internacional, fundada em 1946, que possui como objetivo o desenvolvimento e a publicação de normas técnicas para aplicação mundial. Em 1987, o Comitê Técnico TC 176 (Garantia da Qualidade) desenvolveu as normas da série 9000 (19), que são diversos guias que se referem ao gerenciamento da qualidade e do ambiente da empresa e, de modo geral, possuem foco no atendimento da garantia da qualidade para os clientes.

As normas ISO 9000 representam o resultado do esforço de diversos países que buscaram interpretar e dar forma ao conceito de Gestão da Qualidade. A ISO 9000 descreve diversos aspectos a serem considerados em um gerenciamento da qualidade (33). O guia direciona e dita ferramentas a serem utilizadas a fim de garantir que os produtos e serviços prestados estejam sempre de acordo com os requisitos dos clientes e para que a qualidade seja constantemente melhorada. Foram criados 
mecanismos completos de auditorias internas e externas que contribuíram para esse sistema. A ISO 9001 é uma certificação utilizada por milhares de empresas ao redor do mundo. Essa certificação determina os critérios de qualidade de um sistema de gerenciamento. A versão publicada em 2008 aprofunda a compreensão sobre diversas dimensões que impactam a gestão das organizações com foco na satisfação dos clientes, e por isso, possui bastante visibilidade (34). Ter um certificado ISO 9000 significa que uma empresa possui um sistema de gestão e garantia da qualidade totalmente comprometido com a satisfação do cliente, em todos os níveis, e que, além disso, atende aos requisitos de uma norma internacional.

Para que uma empresa possua certificação ISO, a mesma deve passar pelo processo de acreditação, por meio da contratação de uma empresa certificadora credenciada (19).

\section{REFERÊNCIAS}

1. Calarge FA, Satolo EG, Satolo LF. Aplicação do sistema de gestão da qualidade BPF (boas práticas de fabricação) na indústria de produtos farmacêuticos veterinários. Gest. Prod. 2007; 14 (2):379-392. DOI: 10.1590/S0104530X2007000200014.

2. FIP. International Pharmaceutical Federation Congress, 69. Barcelona; 1999.

3. Isaac G, Rajendran CS, Anantharaman RN. Significance of quality certification: the case of the software industry in India. Quality M J, 2004;11 (1): 8-32. DOI: 10.7763/ IJIMT.2011.V2.132

4. BRASIL. Ministério da Saúde. Resolução nº 17, de 16 de abril de 2010. ed. Brasília (DF); 2010.

5. BRASIL. Ministério da Saúde. Resolução nº 33, de 05 de agosto de 2015. ed. Brasília (DF); 2015.

6. BRASIL. Ministério da Saúde. Resolução nº 13, de 14 de março de 2013. ed. Brasília (DF); 2013.

7. BRASIL. Ministério da Saúde. Resolução n ${ }^{\circ} 63$, de 18 de dezembro de 2009. ed. Brasília (DF); 2009.

8. BRASIL. Ministério da Saúde. Resolução n ${ }^{\circ} 69$, de 01 de outubro de 2008. ed. Brasília (DF); 2008.

9. BRASIL. Ministério da Saúde. Resolução no 9 , de 4 de março de 2010. ed. Brasília (DF); 2010.

10. World Health Organization. Quality assurance of pharmaceuticals guidelines. World Health Organization; 2004.

11. International Conference on Harmonization. Disponível em: < http://www.ich.org/home.html $>$.

12. International conference on harmonization of technical requirements for registration of pharmaceuticals for

\section{CONCLUSÃO}

Existem diversos guias oficiais e publicações que auxiliam na prática de um sistema de gestão da qualidade na indústria. Após reunir os principais guias e práticas relacionadas aos indicadores de qualidade em indústria farmacêutica, foi possível perceber que as publicações disponíveis explicam detalhadamente os guias oficiais, entretanto a aplicação em si e seus resultados, como estudo de casos, têm pouco ou nenhum relato, uma vez que dentro das pesquisa realizada não foram encontradas publicações sobre esse tema, dificultando assim, exemplificar melhor como a essas estratégias influenciam positivamente no gerenciamento das indústrias farmacêuticas. Dessa forma, espera-se que este trabalho contribua para ampliar a divulgação dos indicadores da qualidade na indústria farmacêutica. human use. Guidance for Industry Q10 Pharmaceutical Quality System. 2009.

13. International conference on harmonization of technical requirements for registration of pharmaceuticals for human use. Guidance for Industry Q8 Pharmaceutical Development. 2005.

14. International conference on harmonization of technical requirements for registration of pharmaceuticals for human use. Guideline Q9 on Risck Management. 2009.

15. ANVISA. Anvisa é novo membro do ICH. Disponível em: <http://portal.anvisa.gov.br/rss///asset_publisher/ Zk4q6UQCj9Pn/content/ com-o-inicio-da-reforma-doich-a/219201 ?inheritRedirect=false $>$. Acesso em: 08 out. 2017

16. U.S. Food and Drug Administration. Disponível em: < www.fda.gov/>.

17. U.S Food and Drug Administration. Pharmaceutical cGMPs for the 21st Century - A risck based approach. ed. Rockville: U.S. Department of Health and Human Services; 2002.

18. U.S Food and Drug Administration. Quality Systems Approach to Pharmaceutical cGMP Regulations. ed. Rockville: U.S. Department of Health and Human Services; 2006.

19. ISO. International organization for standardization. Disponível em: $<\mathrm{http}: / / \mathrm{www}$.iso.org $>$.

20. Griffith E. Risk management programs for the pharmaceutical industry. Fujitsu Consulting; 2004.

21. Moraes G. Sistema de Gestão de Riscos - Princípios e Diretrizes. $1^{\mathrm{a}}$. ed. Rio de Janeiro. 2010. 


\section{Ungarma}

22. Rathore AS, Winkle H. Quality by design for biopharmaceuticals. Nat Biotechnol, 2009. 27 (1): 26-34. DOI: $10.1038 / \mathrm{nbt} 0109-26$.

23. Rathore AS, Sharma A, Chillin D. Applying Process Analytical Technology to Biotech Unit Operations. BioPharm Int, 2006; 19: 48-57. DOI: 10.1002/ JCTB. 4447.

24. Rathore AS, Branning R, Cecchini D. Quality: Design Space for Biotech Products. BioPharm Int, 2007. 20: 36-40.

25. Carvalho MM, Paladini EP. (Org.). Gestão da qualidade: teoria e casos. 2. ed. Rio de Janeiro: Elsevier: ABEPRO. 2012.

26. Moura H. Gerenciamento da Qualidade. In: Carvalho M, Paladini E. (Org.). Gestão da Qualidade: Teoria e Caos. Elsevier. 2012.

27. Goeke RJ, Offodile OF. Forecasting management philosophy life cycles: a comparative study of Six Sigma and TQM. Quality M J, 2005. 12 (2): 34-46.

28. Stamatis DH. Six Sigma and beyond-foundation of excellent performance. 2002.
29. Ishikawa K. Controle de qualidade total à maneira japonesa. $6^{a}$ ed. Campus. 1997.

30. Womack J, Jones DT, Roos D. The Machine that Changed the World: The Story of Lean Production. Harper Collins Publisher. 1990.

31. Nave D. How to Compare Six Sigma, Lean and the Theory of Constraints - A framework for choosing what's best for your organization. Quality ProgressMarch: American society for quality. 2002.

32. Bianco M, Salerno MS. Como o TQM opera e o que muda nas empresas? Um estudo a partir de empresas líderes no Brasil. Gest. Prod., 2001. 8 (1): 56-67. DOI: 10.1590/S0104-530X2001000100005.

33. SO. International organization for standardization. ISO 9000:2015: Quality management systems - Fundamentals and vocabulary. ed. ISO: 2015.

34. ISO. International organization for standardization. ISO 9001:2008: Quality management systems - requirements. ed. ISO: 2008. 